\title{
Interactions of children and young adults using large-scale elastic displays
}

\author{
Franceli L. Cibrian, Deysi Ortega, Judith Ley, Hugo Rodríguez, Monica \\ Tentori
}

Published: 30 November 2020

\begin{abstract}
Elastic displays provide a unique and intuitive interaction and could be deployed at large-scale. As an emerging technology, open questions about the benefits large-scale elastic displays offer over rigid displays and their potential application to our everyday lives. In this paper, we present an overview of a 4 -year project. First, we describe the development of a large-scale elastic display, called BendableSound. Second, we explain the results of a laboratory study, showing the elastic display has a better user and sensory experience than a rigid one. Third, we describe the results of two deployment studies showing how BendableSound could support the therapeutic practices of children with autism and the early development of toddlers. We close discussing open challenges to study the untapped potential of elastic displays in pervasive computing.
\end{abstract}

\section{Keywords}

Elastic-displays; Deformable Surfaces; Autism; Children

\section{Introduction}

Elastic displays are a type of deformable display that allows temporal deformations [15], allowing users to use gestures and explore novel ways of interaction that might enable them to use the twenty-three degrees of freedom they have at their fingertips [32] Elastics displays extend touch-based interactions, including an extra dimension, by enabling users to vary the amount of pressure used when touching the surface [26]. The size of the surface [23] is one factor in classifying the type of deformable displays. Largescale displays are those bigger than four feet [7].

As an emerging technology, most of the current studies on elastic displays have focused on evaluating the performance of such displays [6,28]. However, evaluations of large-scale elastic displays are scarce. Descriptions of such real-life deployments showing the value of elastic displays are needed to better

\footnotetext{
Cibrian, Franceli L.,

Chapman University

cibrian@chapman.edu
}

Ortega, D., Rodríguez, H., Tentori, M.

CICESE,

deysukiz@gmail.com,hugo.rgz.a@gmail.com,mtentori@cicese.mx

Ley, J.

Universidad Carlos III de Madrid, Spain.

jley@inf.uc3m.es understand the interaction experience of large-scale elastic displays and their potential value to users. Such empirical evidence could help pervasive computing researchers to improve the design and development of large-scale elastic displays.

This papers aim to provide an overview of a research project on elastic displays to provide empirical evidence about potential differences in the user and the sensory experience when using a large-scale elastic display vs. a rigid one; and how those differences and advantages can be used in two concrete scenarios deployed in real-life situations.

\section{Related work}

\subsection{Deformable and Elastic Surfaces}

One of the first research questions researchers have explored is to uncover what are the best "technology" materials appropriate for the development of elastic displays [12,33]. For example, Troiano et al. [33] conducted three workshops with nine musicians studying different deformable objects appropriate for music performances. Deformable objects included a spandex fabric, a foam cube, a ballshaped sponge, clay, and a plastic cylinder. Their results indicate the fabric was perceived highly deformable, intuitive, easy to learn and fun, and better enable musicians to add effects to sounds.

Others have studied the development and performance of elastic displays [6,28] to support navigation [36], and manipulation of physical [26] and multimedia [5] information. For example, TableHop [28], is a small-scale elastic tabletop using projections to physicalize earthquake data through $3 \mathrm{D}$ animations. TableHop uses overlapping transparent electrodes that vibrate when the earthquake is shaking the ground. Performance evaluation of TableHop indicates the fabric presents hysteresis when being bend, calibration is paramount to ensure its usage under optimal conditions, and higher voltages are required for larger vibrations. A pilot study with six young adults using TableTop shows the tactile feedback was appropriate as users easily perceived changes in the vibrations.

Similarly, others have explored how to uncover novel potential interactions with elastic displays [32]. For example, a laboratory study where 17 young adults (with experience with touch-based displays like tablets, smartphones) used a small-scale elastic display to perform predefined touch-based interactions, like displacing and rotating objects. The results of this study show that the affordances of elastic displays enable users to interact differently with the canvas by pulling it, pushing it, or twisting it [32]. This body of work shows that the selection of materials must be related to the performed activity (in the case of [33] music) 


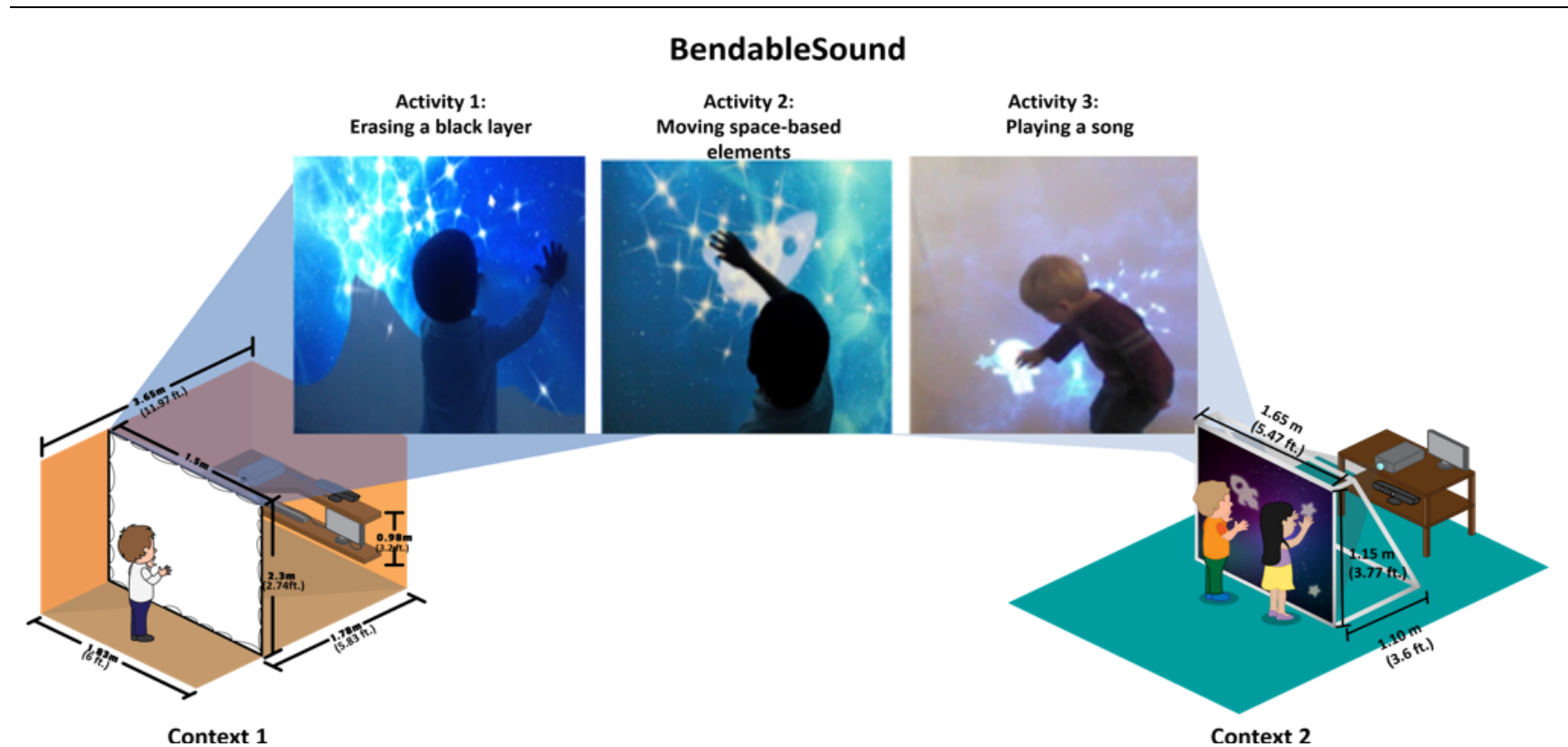

Figure 1. Setting up BendableSound inside the school-clinic of children with autism (left-bottom), and inside the classroom of toddlers (right-bottom). Children interacting with BendableSound. From top-left to top-right: A child taps and touches the fabric removing the black layer covering the space nebula; one child moving a rocket while making harmonic sounds; and a child playing a song by following the astronaut.

Spandex fabric is an appropriate material for those interested in enhancing users' interactions with music.

\subsection{Musical Interfaces to Support Neurodiverse Users and Child Development}

Musical interfaces can be used to exchange music between the user and an interactive interface. They include digital musical instruments, augmented traditional instruments, or interactive installations [17,18,25,35]. For example, Andantino [35] is a piano augmented with a projection of animated figures representing musical elements such as sound quality, moods in the music or harmonies. The aim of Andantino is to provide an enjoyable music learning environment for children.

Other musical interfaces have been proposed to support individuals with motor and cognitive disabilities $[4,14,16]$. For example, MINWii [4] is a serious game for patients with dementia that allows them to play different songs. A deployment study of the use of MINWii shows that combining tangible and gestural interactions ease the process of playing music for patients with cognitive impairments.

These workS shows that musical interfaces are appropriate for children and individuals with disabilities. On the other hand, deformable and elastic display may facilitate interaction with music. However, to our knowledge, there are no studies comparing the potential differences between rigid and elastic display and how the advantage or elastic display can be used in real-life scenarios.

\section{BendableSound: Developing a large-scale elastic display}

BendableSound is a large-scale elastic display that allows users to make music when tapping and touching on top of the canvas (Figure 1) (for more details about the design process, we referred the reader to $[8,9]$ ). Users can play the sounds of different musical instruments. Musical notes are arranged from low to high pitch in an ascending major scale on the vertical axis. BendableSound displays a $3 \mathrm{D}$ background showing an animation of a nebula with translucent space-based elements, like stars and planets, randomly appearing on top of the fabric.

BendableSound is a multi-touch system using the Kinect sensor, two audio speakers, and an ultra-short throw projector placed behind a spandex fabric (Figure 1). The Kinect sensor, the speakers and the projector are connected to a PC. BendableSound uses the TSPS library to read Kinect's data and infer users' interactions. We used the Processing language and a 3D shader to develop multimedia interfaces (Figure 1). We used the OSC protocol to integrate the Kinect's data and multimedia interfaces.

\section{Pilot study: potential differences between rigid and elastic displays}

We conducted a within-subjects pilot study aimed to evaluate differences in the user and the sensory experience when using a large-scale elastic display in contrast to a rigid one.

Twenty young adults participated in the study (16 men and 4 women aged between 23 and 30 years old). We recruited the participants with flyers placed in a Research Center in Mexico. As inclusion criteria, participants should have experience with touchbased surfaces, like smartphones, and do not exhibit any sensory issues. Participants used BendableSound with the same animation and dimensions in two conditions: using a large-scale, elastic display (C1) and rigid display (C2) (Figure 2).

Participants randomly rotated between the two conditions conducting two activities lasting for around one minute each. In the first activity, participants practiced gestures like swiping, pinching, pushing, and tapping. For the second activity, participants were able to interact in a free-way. At the end of each condition, 


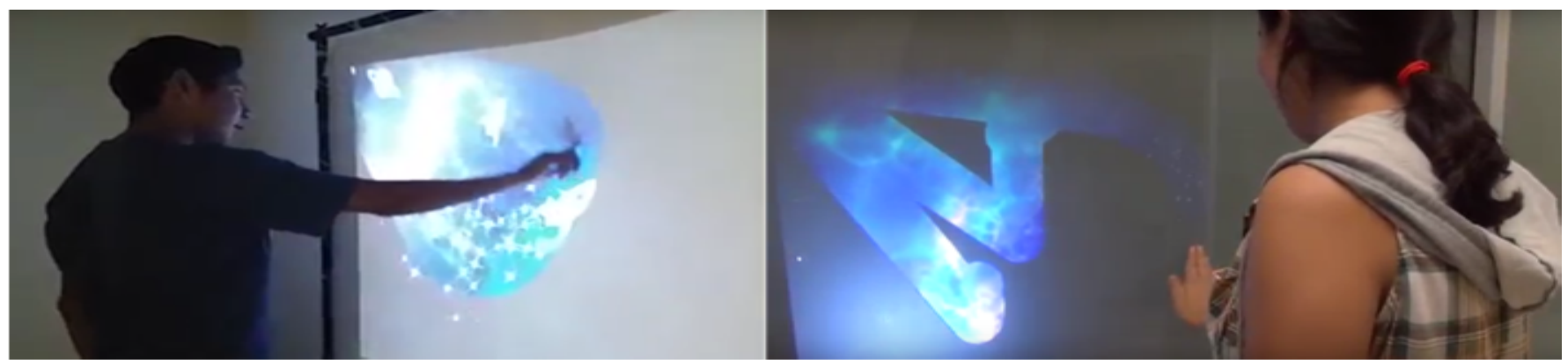

Figure 2. Young adults using BendableSound in two conditions: using the elastic display (left) and a rigid display (right)

participants answered the User Experience Questionnaire (UEQ) ${ }^{1}$ [22] and the sensory experience questionnaire ${ }^{2}[19,24]$. To compute the questionnaire scores, we scored each item using a scale from 3 to +3 , and we calculated the average score for all the items. We used a t-test to compare the results of each participant under each condition.

\subsection{Results}

Overall, our laboratory study shows participants found the largescale elastic display with a better user and sensory experience than the rigid display.

\subsubsection{User experience}

$95 \%$ of participants preferred to use the elastic display than the rigid display; as the elastic display provided a better user experience than the rigid one (Figure 3, top). $85 \%$ of participants found the elastic display more attractive $(\mathrm{p}=0.005), 80 \%$ perceived it more stimulating $(\mathrm{p}=0.012)$ and $70 \%$ more original $(\mathrm{p}=0.017)$. This result could be because participants found more enjoyable the affordances provided by the elastic display allowing them to interact with the display and be more creative more naturally, but also, this preference can be given the novelty of interactive with an elastic fabric.

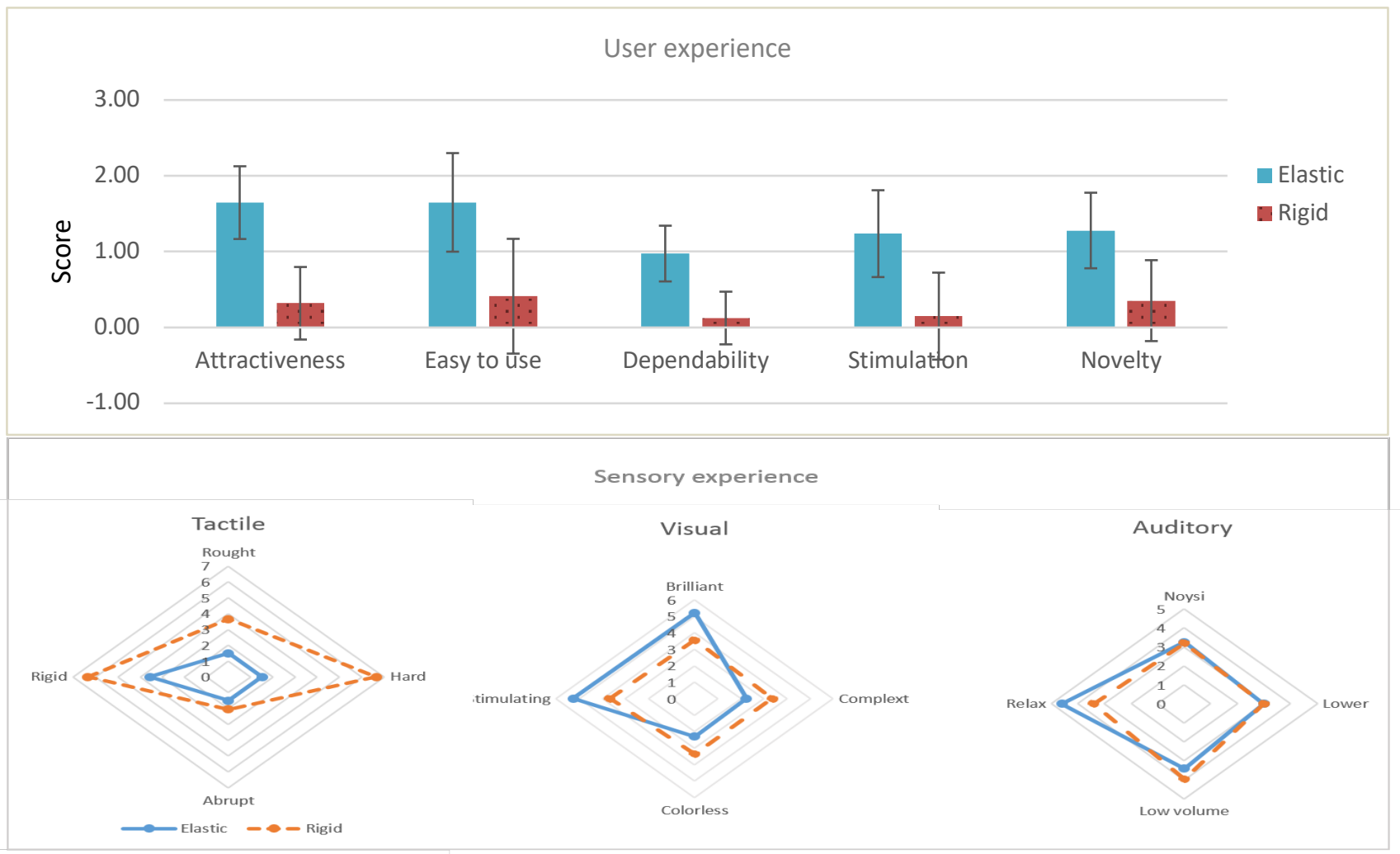

Figure 3. Average of participants' scores to UEQ survey (top). Results of the tactile, visual and auditory stimuli (bottom). As expected, the area of the figures was very different.

1 The UEQ is a 7-point bipolar scale questionnaire measuring attractiveness, perspicuity, efficiency, dependability stimulation, and novelty,
${ }^{2}$ The sensory experience is 7-point bipolar scale survey evaluating if the display invites the user to touch it, and measures user's perception to visual, tactile, auditory stimuli. 
$85 \%$ of participants perceived the activities performed with the elastic display were easier $(p=0.02)$ as participants could feel more fluency when moving the digital elements on the canvas. $85 \%$ of participants found the elastic display more reliable $(p=0.0001)$ as the rigid display was perceived more fragile than the elastic display, especially when touching it too strongly.

\subsubsection{Sensory experience}

$80 \%$ of participants perceived the elastic display invites them to manipulate the animations, whereas $45 \%$ of participants perceived the same with the rigid display (elastic; rigid Avg. $=0.85$ out 3; $\mathrm{p}=0.003$ ). This result could be explained as participants perceived the properties of the fabric to be more appealing when seeing them or touching them. $75 \%$ of participants found the elastic display feels "good" when they touch it; in contrast with the rigid display that only $25 \%$ of the participants felt the same way (elastic Avg. $=1.18$; rigid Avg. $=0.15 ; \mathrm{p}=0.003)$. We attribute these results to the consistency of the multisensory stimuli that facilitate participants to match the visual stimuli more easily to both the audible and the tactile sensations.

$95 \%$ of participants expressed that the elastic display provides a better tactile sensation than the rigid display. $80 \%$ of participants describe the elastic display as soft, $50 \%$ perceived it as flexible and more pleasant to touch (Figure 3 bottom-left). Participants perceived a different sensory experience when using the elastic display in contrast to the rigid one. As the visual and auditory stimuli were exactly the same for both displays, we did not expect a variation in the results; however, more than half users perceived the elastic display as being brighter (50\%), with simpler visualizations $(75 \%)$, more simulating $(55 \%$, Figure 3 , bottomcenter) and more relaxing (60\%, Figure 3 , bottom-right). These results show that the elastic display could alter the sensory experience perceived by participants as it might have better perceptual integration.

\subsection{Lesson Learned}

Given the novelty interaction experience of the elastic display, the users' experience was highlight superior to the rigid display. As expected, the tactile experience was very different between the two surfaces, but this also affects the auditory and visual experience, as young adults found it more stimulating and relaxed. These two aspects are important when design for therapeutic or playful interaction with children. Thus, we decided to explore the potential value of the large-scale elastic display in those scenarios.

\section{Deployment studies}

Here we present an overview of two studies exploring the potential value of large-scale elastic displays for children.

\subsection{Study 1: Supporting Therapeutic Practices of Children with Autism}

Autism is estimated to affect 1 in 59 children worldwide [3], and 1 in 115 [13] is estimated in Mexico. Autism is a neurodevelopmental disorder associated with impairments in attention, social interaction, and behavior [1].

\footnotetext{
${ }^{3}$ Events where the child, without the intervention of the psychologist or teacher, spontaneously initiates an interaction or change the interaction mode (e.g., the child is using one finger and then he is using both hands)
}

Music Therapy is increasingly being used to support the sensorimotor regulation of individuals with autism with promising clinical results [21]. Music-therapy, heavily relying on the voluntary use of musical instruments, is being used to support children with severe autism with promising clinical results [31]. Music-therapy encourages the interactive use of music and their musical elements [31].

We followed an iterative user-centered design methodology to adapt BendableSound and design two activities supporting musictherapy sessions (for more details about the design process, we referred the reader to [8]). We develop two activities. In the first activity, children must erase a black layer covering the animation of nebulas (Figure 1 top-right). In the second activity, children move space-based elements randomly appearing in the fabric canvas that will reproduce sounds when touched or moved (Figure 1 top-center).

We deployed this version of BendableSound in a school-clinic attending children with autism in the Northwest of Mexico. Twenty-four children and one psychologist attended a 5-minute music-therapy session, randomly rotating between using a keyboard piano and interacting with BendableSound [8].

Teachers perceived the installation safe but not portable enough to move the prototype around the clinic premises. For each condition, we specifically measured voluntarily interactions ${ }^{3}$ and the amount of time children were focused during the therapy. We conducted a special analysis to uncover novel interactions children exhibited when using the elastic display.

Our results show children with autism spend $7.11 \%$ more time focused during therapy sessions $(p=0.02)$ [8] and doubled the number of times they voluntarily initiated interactions when using the elastic display than when using a keyboard piano $(p=0.04$; Figure 4 left-top). Furthermore, although most of the time, children with autism were interacting with the elastic display in a typical manner by tapping or swiping around the canvas, $5 \%$ of the therapy time children with autism explored the use of different parts of their body. During this time, $58 \%$ of the participants used their head, back, or feet to bend the surface (Figure 4 left-bottom).

This result shows the affordances of the elastic display encouraged children with autism to uncover new ways of interaction, even though children with autism are very structured and struggle with new experiences. Full-body interactions could support sensory therapies enabling children to more easily rub against their body a broad range of textures.

\subsubsection{Lesson learned}

This project shows BendableSound successfully supports musictherapy sessions and excels traditional tools currently being used during music-therapy with numerous educational benefits related to cognitive, sensory, and motor skills [8] (for more details about the impact on coordination skills for children with autism, see [9]).

Moreover, the multisensory experience was well accepted for children with attention, social and behavioral differences. Those skills are learned since the early stages of life. Then as a second approach, we use BendableSound to support early development of toddlers. 


\section{Deployment studies results}

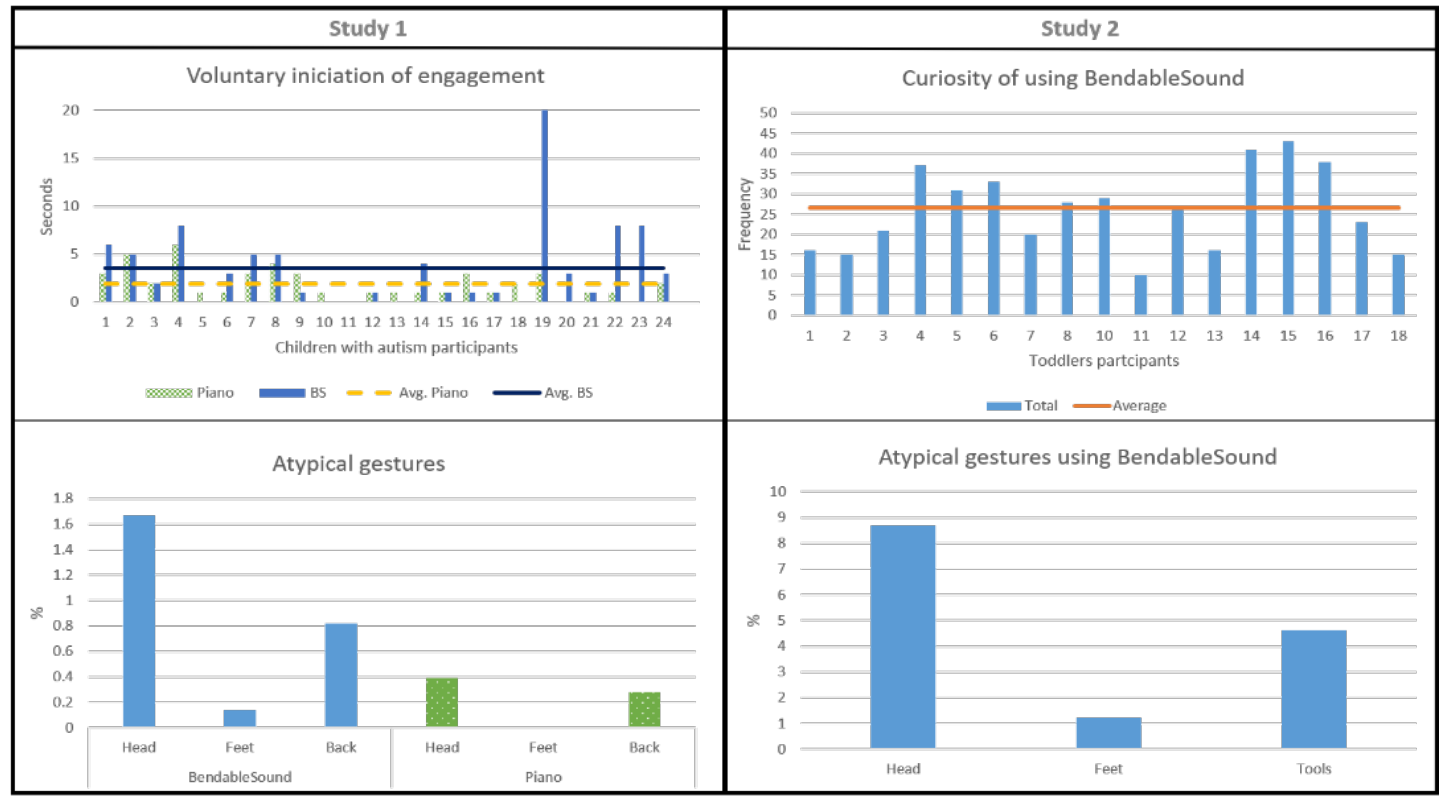

Figure 4. Study 1: Frequency of voluntary initiation of engagement (i.e., events where the child, without the intervention of the psychologist or teacher, spontaneously initiates an interaction or change the interaction model ) that participants experienced when using piano against using BendableSound (BS) (left top). Percent of time that that children with autism perform a gesture (push, or swipe) using different part of their body during the use of BendableSound (left bottom). Study 2: Frequency of curiosity (i.e.,events where the child, without the intervention of the teacher, spontaneously play with BendableSound) (right top). Percent of time that toddlers perform a gesture (push, or swipe) using different part of their body or a tool during the use of BendableSound (right bottom).

\subsection{Study 2: Early Development of Toddlers}

Free play is a voluntary, spontaneous, engaging, pleasurable, and a collaborative activity [29] that is important in any early development program as toddlers learn from their playing experiences. Designing activities to be used during free playtime for toddlers is not an easy task [20]. Free play activities should be interesting, sturdy, age-appropriate, gender-neutral, and arranged to encourage independence, curiosity, and initiative in learning [20].

In this study, we explored if BendableSound was appropriate to support the free playtime in toddler classrooms [11]. Following a participatory approach to meet the needs of toddlers, we included one activity to the previous design of BendableSound. In the third activity, an astronaut appears to provide children with guidance on how to play a song by touching a blinking star [11] (Figure 1 topleft).

During nine weeks, 22 toddlers and 5 teachers from an early education center in the United Stated at Southern California used the new version of BendableSound twice a week during their freeplay time (1.5 hr. per day) [11]. Teachers demanded supervision for toddlers when playing with BendableSound.

Every two weeks, we conducted a focus group with teachers. For 12 days, we video-recorded toddlers' interactions. We quantified $15 \%$ of the recorded videos for the 18 toddlers who consented to video-recording. As in our previous study, we quantified "atypical" interactions, curiosity, an initiative in learning.

Overall, our results show that BendableSound was successfully adopted and integrated in toddler classrooms. Toddlers were highly motivated when playing with BendableSound, they rapidly learned how to use it, and most of the time, they played in teams [11].

We observed that $94 \%$ of toddlers decided to use the elastic display interacting "atypically" by either using their full body (9.91\%) or including tangible objects (4.61\%) (Figure 4, right-bottom). We observed these "atypical" interactions happen for around $15 \%$ of the time during free-play time. These results might indicate that the use of different materials to develop screen-like interfaces could help toddlers envision novel ways of interaction.

Our quantitative results confirm that BendableSound supports the curiosity and initiative in learning, as $94 \%$ of the toddlers exhibited, on average, 26.5 "atypical" interactions without needing help from teachers (Figure 4 right-top).

\subsubsection{Lesson learned}

In the second study, we learned that BendableSound could be used as a free play activity similar to a toy but with numerous educational benefits related to self-regulation, socialization, cognitive, and motor skills [11].

Overall, in the pilot-study and the two deployment studies conducted, we found open challenges and research opportunities mainly in the technology point of view.

\section{Open challenges}

The studies we conducted revealed two main challenges that needed to be addressed to provide a richer user interaction experience with elastic displays and facilitate their development. 


\subsection{Increasing Portability and Robustness}

Research around elastic displays is still in its early stages. Deployment studies are scarce, and prototypes are still very difficult to develop and deploy. Most prototypes require a very complex set up and are highly fragile.

Our studies show that there is a trade-off between portability and robustness. While our installation in the autism clinic proved to be robust as it was heavily customized to the space, users regret the lack of opportunities to move the installation. Intending to build a more portable solution when installing our prototype in toddler classrooms, we used a frame similar to a soccer goal that was independent of the infrastructure available in the classroom (Figure 1). However, this installation was perceived to be unsafe, resulting in constant supervision of the children playing with the elastic display. We regret the lack of advances in technology that enable us to come up with a set up that is truly standalone, portable and easy to deploy. Overall, the development and deployment of elastic surfaces are heavily limited due to the lack of advances in the hardware currently uses for projection and sensing.

From a sensing point of view, there are limited solutions that incorporate sensors directly into the fabric [6,28]. Current technology using the fabric's threads as sensors is too experimental and hardly scalable. Elastic displays are more complex as threads loose resistivity decreasing the precision for detecting users' gestures when deployed at large-scale. In addition, the lifespan of a fabric is very short. The long-term usage causes holes and thread breakages affecting sensing precision. Other elastic surfaces have used optical sensors and 3D cameras, however, the camera must be placed at least $1.5 \mathrm{ft}$. from the fabric making the prototype increasingly wider.

From a visualization point of view, multimedia projectors have become smaller and more portable and could be easily integrated into a piece of fabric. However, there are too expensive to be used "in the wild." Also, multimedia projectors still serve as "dumb terminals" needing a computer to run all the multimedia processing. Embedded computers like Raspberry Pi could facilitate the portability of the software with dedicated functions in small and powerful hardware and could be a potential solution to integrate a small ultra-short multimedia projector with embedded displays that could facilitate the development of elastic displays.

\subsection{Interaction Sensing Tools Invariant to Deformation}

Large-scale elastic displays are highly deformable, creating slopes or valleys around the surface, making it very difficult to detect where the user is touching, what part of the body he is using to interact with the fabric or discriminate between different gestures. In our second deployment study, when toddlers collectively used BendableSound, the sensing library that we used was not precise enough when trying to detect when two or more children touched the fabric at a very close distance. After a time-consuming calibration process, optical indirect sensing solutions are sufficient for detecting individual touch-based interactions. Open questions remain as to develop more robust gesture recognition algorithms that can recognize various deformations more efficiently and when being used collectively.

Current solutions to overcome these challenges could involve the use of an array of heterogeneous sensors combining, for example, several Kinect cameras and leap motion sensors placed in front, behind, and on top of the fabric. Having multiple sensors may overcome challenges associated with occlusion and increase the resolution at a finger level. However, these solutions are not scalable and increase the cost of the installation.

The development of new hardware that requires little maintenance that could be unnoticed by users and algorithms that provide robust and efficient detection invariant to deformation are urgently needed.

\section{Future work}

Although several challenges lay ahead in the design, development, and evaluation of elastic displays, the results from our studies have motivated us to continue working on this domain. We used our lessons learned from our studies to redesign BendableSound. We designed a new set of activities to support children with autism when practicing movements that require variations in strength [10]. We are currently testing the efficacy of using this new version of BendableSound as a therapeutic intervention to improve the selfregulation of the strength of children with autism. Here we outline other future projects in this area.

\subsection{Collaboration Mechanisms for Elastic Displays}

Our results indicate that users wanted to use large-scale elastic displays collectively [11]. However, the current version of our prototype does not take into account collaboration mechanisms. Novel content design and interactions that successfully promote social interactions could help us to understand the design space of elastic displays in social settings. Activities that encourage both synchronous and asynchronous coordination for elastic displays are needed. Social interactions are both important for therapeutic interventions and have been heavily limited in public spaces. Primarily because collective experiences are hard to achieve, but also because users no longer have a medium that facilitates them. So, there is an untapped potential to study if the playful interaction provided by elastic displays could serve as an "ice-breaker" that might lead to richer collective experiences. Preliminary efforts on this direction have been made using different light markers to identify each participant [34].

\subsection{Touch and Gestural Analysis for the Detection of Behavioral Markers}

Our results indicate that users uncovered novel ways of interaction with large-scale elastic displays[8] [11]. The analysis of users' interactions could help us to infer the identity of the user or deviations in the patterns of their interaction, as others have pointed out $[2,27]$. For example, studies have found that children with autism interact differently when using rigid small-scale displays by using more force when pressing the surface and tapping in a faster way [2]. Elastic displays provide better affordances to more easily discover and classify tapping behaviors [26]. The development of appropriate machine learning techniques to identify such behaviors could allow the identification of behavioral markers to help with the early detection of autism and related disorders.

Similarly, 3D interactions have been identified as capable of detecting the variations in the position of hands to infer affective states [27]. Elastic displays using 3D descriptors of hand movements and finger pressure could help to more easily identify descriptors of the expressive interactions, like tiredness, tension, pain, and satisfaction [33].

\subsection{Sonification of Movements}

Our results show that elastic displays could alter the sensory experience. Researchers have been studied sonification techniques 
varying the structure of sounds to alter the body perception of movements [30]. For example, a study with patients with chronic pain found that varying the musical structure as audible feedback could help patients to stretch beyond their movement range [30]. So there is an untapped potential to study the musical structure that is more appropriate to guide the movement of users, and how such auditory feedback could be used in tandem with the tactile affordances offered by elastic-displays.

The lessons we learned from this project might benefit other researchers interested in understanding the design space of elastic displays. Many of the open challenges we have discussed here could also facilitate the development of deformable surfaces more broadly.

\section{Acknowledgment}

We thank all the participants enrolled in the studies and the researchers and the reviewers who provide helpful comments on previous versions of this document. We particularly thank Nadir Weible for their support on the Study 2. We also thank CONACYT for students' fellowships

\section{References}

[1] American Psychiatric Association. 2013. Diagnostic and statistical manual of mental disorders (DSM-5®). American Psychiatric Association, Washington, DC. London, England. https://doi.org/10.1176/appi.books.9780890425596.744053

[2] Anzulewicz Anna, Sobota Krzysztof, and Delafield-Butt, Jonathan T. 2016. Toward the Autism Motor Signature: Gesture patterns during smart tablet gameplay identify children with autism. Scientific Reports 6, 31107: 1-13. https://doi.org/10.1038/srep31107

[3] Baio Jon, Wiggins Lisa, Christensen Deborah L., Maenner Matthew J, Daniels Julie, Warren Zachary,... and Dowling Nicole F. 2018. Prevalence of Autism Spectrum Disorder Among Children Aged 8 Years - Autism and Developmental Disabilities Monitoring Network, 11 Sites, United States, 2014. MMWR Surveill Summ 67, 6: 1-23. https://doi.org/http://dx.doi.org/10.15585/mmwr.ss6706a1

[4] Boulay Mélodie, Benveniste Samuel, Boespflug Sandra, Jouvelot Pierre, and Rigaud Anne Sophie. 2011. A pilot usability study of MINWii, a music therapy game for demented patients. Technology and Health Care 19, 4: 233246. https://doi.org/10.3233/THC-2011-0628

[5] Cassinelli Alvaro and Ishikawa Masatoshi. 2005. Khronos projector. In ACM SIGGRAPH 2005 Emerging technologies on - SIGGRAPH '05, 10. https://doi.org/10.1145/1187297.1187308

[6] Chang Angela and Ishii Hiroshi. 2007. Zstretch: a stretchy fabric music controller. NIME'07 Proceedings of the 2007 international conference on New interfaces for musical expression: $46-49$. https://doi.org/http://doi.acm.org/10.1145/1279740.1279746

[7] Chung Seung Eun and Ryoo Han Young. 2016. Relationships between Flexible Display's Form Factors and its User Experience Types. International Journal of Multimedia and Ubiquitous Engineering 11, 10: 245-252. https://doi.org/10.14257/ijmue.2016.11.10.23

[8] Cibrian, F. L., Pena, O., Ortega, D. \& Tentori. 2017. BendableSound: Designing a Fabric-based Interactive
Surface to Support Neurologic Music Therapy sessions for children with severe autism. Int. J. Hum. Comput. Stud 107: 22-37. https://doi.org/https://doi.org/10.1016/j.ijhcs.2017.05.003

[9] Cibrian, F. L., Madrigal, M., Avelais, M. and Tentori M.. 2020. Supporting coordination of children with ASD using neurological music therapy: A pilot randomized control trial comparing an elastic touch-display with tambourines. Research in Developmental Disabilities.

[10] Cibrian, F.L. Pena, Oscar, Vazquez, Vianey, Cardenas, Carlos and Tentori, M. 2016. Designing a deformable musical surface for children with autism. In Proceedings of the 2016 ACM International Joint Conference on Pervasive and Ubiquitous Computing: Adjunct - UbiComp '16, 977982. https://doi.org/10.1145/2968219.2968262

[11] Cibrian, F.L., Weibel Nadir, and Tentori Monica. 2016. Collective use of a fabric-based interactive surface to support early development in toddler classrooms. In Proceedings of the 2016 ACM International Joint Conference on Pervasive and Ubiquitous Computing - UbiComp '16, 328-339. https://doi.org/10.1145/2971648.2971695

[12] Follmer Sean, Leithinger Daniel, Olwal Alex, Cheng Nadia, and Ishii Hiroshi. 2012. Jamming user interfaces: programmable particle stiffness and sensing for malleable and shape-changing devices. In Proceedings of the 25th annual ACM symposium on User interface software and technology - UIST '12, 519-528.

https://doi.org/10.1145/2380116.2380181

[13] Fombonne Eric, Marcin Carlos, Manero Ana Cecilia, Bruno Ruth, Diaz Christian, Michele Villalobos,... and Nealy Benjamin. 2016. Prevalence of Autism Spectrum Disorders in Guanajuato, Mexico: The Leon survey. Journal of Autism and Developmental Disorders 46, 5: 1669-1685. https://doi.org/10.1007/s10803-016-2696-6

[14] Gorman Mikhail, Lahav Amir, Saltzman Elliot, and Betke Margrit. 2007. A camera-based music-making tool for physical rehabilitation. Computer Music Journal 31, 2: 3953. https://doi.org/10.1162/comj.2007.31.2.39

[15] Grunder Thomas, Kammer Dietrich, Brade Marius, and Groh Rainer. 2013. Towards a design space for elastic displays. In CM SIGCHI Conference on Human Factors in Computing Systems - Workshop on Displays Take New Shape: An Agenda for Interactive Surfaces, 1-4.

[16] Hobbs David and Worthington-Eyre Breanna. 2008. The efficacy of combining augmented reality and music therapy with traditional teaching - Preliminary results. $i$-CREATe 2008 - International Convention on Rehabilitation Engineering and Assistive Technology 2008: 241-244.

[17] Ichino Junko, Pon Aura, Sharlin Ehud, Eagle David, and Carpendale Sheelagh. 2014. Vuzik: the Effect of Large Gesture Interaction on Children's Musical Expression. In Proceedings of the 26th Australian Computer-Human Interaction Conference on Designing Futures the Future of Design, 240-249. https://doi.org/10.1145/2686612.2686649

[18] Jordà Sergi, Geiger Günter, Alonso Marcos, and Kaltenbrunner Martin. 2007. The reacTable: Exploring the Synergy between Live Music Performance and Tabletop Tangible Interfaces. In Proceedings of the 1st international 
conference on Tangible and embedded interaction - TEI '07, 139. https://doi.org/10.1145/1226969.1226998

[19] Klatzky Roberta L. and Peck Joann. 2012. Please touch: Object properties that invite touch. IEEE Transactions on Haptics 5, 2: 139-147. https://doi.org/10.1109/TOH.2011.54

[20] Knopf Herman T and Welsh Kerrie L. 2010. INFANT / TODDLER MATERIALS GUIDE. USC Child Development Research Center.

[21] LaGasse A. B. and Hardy M. W.. 2013. Considering rhythm for sensorimotor regulation in children with autism spectrum disorders. Music Therapy Perspectives 31, 1: 67-77. https://doi.org/10.1093/mtp/31.1.67

[22] Laugwitz Bettina, Held Theo, and Schrepp Martin. 2008. Construction and Evaluation of a User Experience Questionnaire. In HCI and Usability for Education and Work, 63-76. https://doi.org/10.1007/978-3-540-89350-9_6

[23] Lee Sang-su, Lim Youn-kyung, and Lee Kun-Pyo. 2012. Exploring the effects of size on deformable user interfaces. Proceedings of the 14th international conference on Humancomputer interaction with mobile devices and services companion: 89-94. https://doi.org/10.1145/2371664.2371682

[24] Martín Rodrigo, Iseringhausen Julian, Weinmann Michael, and Hullin Matthias B.. 2015. Multimodal perception of material properties. In Proceedings of the ACM SIGGRAPH Symposium on Applied Perception - SAP '15, 33-40. https://doi.org/10.1145/2804408.2804420

[25] Morreale Fabio, De Angeli Antonella, and O’Modhrain Sile. 2014. Musical Interface Design: An Experience-oriented Framework. Proceedings of the International Conference on New Interfaces for Musical Expression: 467-472. Retrieved from http://www.nime.org/proceedings/2014/nime2014_437.pdf

[26] Müller Mathias, Gründer Thomas, and Groh Rainer. 2015. Data exploration on elastic displays using physical metaphors. In $x$ CoAx 2015: Proceedings of the Third Conferenc on Computation, Communication, Aesthetics and $X, 111-124$.

[27] Rivas Jesús, Orihuela-Espina Felipe, Sucar L., Palafox Lorena, Hernández-Franco Jorge, and Bianchi-Berthouze Nadia. 2015. Detecting affective states in virtual rehabilitation. EAI Endorsed Transactions on Pervasive Health and Technology 15, 2: 287-292. https://doi.org/10.4108/icst.pervasivehealth.2015.259250

[28] Sahoo Deepak Ranjan, Hornbæk Kasper, and Subramanian Sriram. 2016. TableHop: an actuated fabric display using transparent electrodes. Proceedings of the $2016 \mathrm{CHI}$
Conference on Human Factors in Computing Systems: $3767-$ 3780. https://doi.org/10.1145/2858036.2858544

[29] Santer Joan, Griffiths Carol, Goodall Deborah L, National Children's Bureau, and Play England. 2007. Free play in early childhood: A literature review. National Children's Bureau National.

[30] Singh Aneesha, Piana Stefano, Pollarolo Davide, Volpe Gualtiero, Varni Giovanna, Tajadura-Jiménez Ana,... and Bianchi-Berthouze Nadia. 2016. Go-with-the-Flow: Tracking, Analysis and Sonification of Movement and Breathing to Build Confidence in Activity Despite Chronic Pain. Human-Computer Interaction 31, 3-4: 335-383. https://doi.org/10.1080/07370024.2015.1085310

[31] Sudha M Srinivasan and Anjana N Bhat. 2013. A review of "music and movement" therapies for children with autism: embodied interventions for multisystem development. Frontiers in integrative neuroscience 7, 22: 1-15. https://doi.org/10.3389/fnint.2013.00022

[32] Troiano Giovanni Maria, Pedersen Esben Warming, and Hornbæk Kasper. 2014. User-defined gestures for elastic, deformable displays. In Proceedings of the 2014 International Working Conference on Advanced Visual Interfaces 1-8. https://doi.org/10.1145/2598153.2598184

[33] Troiano Giovanni Maria, Pedersen Esben Warming, and Hornbæk Kasper. 2015. Deformable Interfaces for Performing Music. In Proceedings of the 33rd Annual ACM Conference on Human Factors in Computing Systems - CHI '15, 377-386. https://doi.org/10.1145/2702123.2702492

[34] Vazquez Vianey, Cibrian Franceli L., and Tentori Monica. 2019. StretchyStars: a multitouch elastic display to support cooperative play among preschoolers. Personal and Ubiquitous Computing 23, 1: 99-115. https://doi.org/10.1007/s00779-018-1179-5

[35] Xiao Xiao, Puentes Pablo, Ackermann Edith, and Ishii Hiroshi. 2016. Andantino. In Proceedings of the The 15th International Conference on Interaction Design and Children - IDC '16, 37-45. https://doi.org/10.1145/2930674.2930689

[36] Yun Kyungwon, Song JunBong, Youn Keehong, Cho Sungmin, and Bang Hyunwoo. 2013. ElaScreen: Exploring Multi-dimensional Data using Elastic Screen. In Proceedings of the extended abstracts of the SIGCHI Conference on Human Factors in Computing Systems (CHI EA '13), 13111316. https://doi.org/10.1145/2468356.2468590

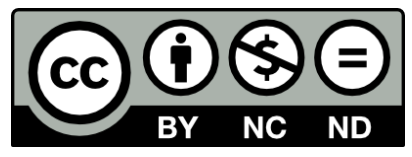

(C) 2020 by the authors. This work is licensed under the Creative Commons AttributionNonCommercial-NoDerivatives 4.0 International License. To view a copy of this license, visit http://creativecommons.org/licenses/by-nc-nd/4.0/ or send a letter to Creative Commons, PO Box 1866, Mountain View, CA 94042, USA. 